\title{
SISTEMATIZAÇÃO DA ASSISTÊNCIA DE ENFERMAGEM PERIOPERATÓRIA NA SEGURANÇA DO PACIENTE: REVISÃO INTEGRATIVA
}

\author{
Systematization of perioperatory nursing assistance in patient safety: an integrative review
}

\author{
Sistematización de la asistencia de enfermería \\ perioperatoria en la seguridad del paciente: revisión integrativa

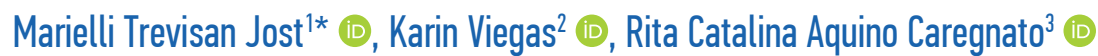

RESUMO: Objetivo: Conhecer a abordagem de artigos científicos sobre a sistematização da assistência de enfermagem perioperatória relacionada à segurança do paciente. Método: Revisão integrativa com buscas nas bases de dados da Biblioteca Virtual em Saúde, da National Library of Medicine (PubMed) e dos periódicos da Coordenação de Aperfeiçoamento de Pessoal de Nível Superior (CAPES). Artigos avaliados conforme o nível de evidência utilizando o instrumento de avaliação proposto pela Joanna Briggs Institute (JBI). Resultados: Identificaram-se dez artigos, sendo oito publicados em periódicos nacionais e dois em internacionais. Os estudos foram classificados conforme níveis de evidência (NE): três com NE 5 e sete com NE 6. Conclusões: Consideram-se poucas as publicações sobre sistematização da assistência de enfermagem perioperatória relacionada à segurança do paciente. Os artigos selecionados apresentam baixo nível de evidência e a abordagem direciona-se à segurança do paciente, restringindo-se à utilização do checklist e implementação da sistematização da assistência de enfermagem perioperatória. Palavras-chave: Assistência perioperatória. Enfermagem perioperatória. Centro cirúrgico. Segurança do paciente.

ABSTRACT: Objective: To know the approach of scientific articles on the systematization of perioperative nursing care related to patient safety. Method: Integrative review with searches in the databases of the Virtual Health Library, National Library of Medicine (PubMed), and the journals of the Coordination of Improvement of Higher Education Personnel (CAPES). Articles were evaluated according to the level of evidence, using the evaluation tool proposed by the Joanna Briggs Institute (JBI). Results: Ten articles were identified, of which eight were published in national journals, and two in international journals. The studies were classified according to levels of evidence (LoE): three with LoE 5 and seven with LoE 6. Conclusions: There are few publications on the systematization of perioperative nursing care related to patient safety. The articles selected present a low level of evidence and the approach is directed towards patient safety, being restricted to the use of the checklist and implementation of the systematization of perioperative nursing care.

Keywords: Perioperative care. Perioperative nursing. Surgical center. Patient safety.

RESUMEN: Objetivo: Conocer el enfoque de los artículos científicos sobre la sistematización de la atención de enfermería perioperatoria relacionada con la seguridad del paciente. Método: Revisión integradora con búsquedas en las bases de datos de la Biblioteca Virtual en Salud, la Biblioteca Nacional de Medicina (PubMed) y las revistas de Coordinación de Mejora del Personal de Educación Superior (CAPES). Los artículos fueron evaluados según el nivel de evidencia, utilizando la herramienta de evaluación propuesta por el Instituto Joanna Briggs (JBI). Resultados: se identificaron diez artículos, de los cuales ocho se publicaron en revistas nacionales y dos en revistas internacionales. Los estudios se clasificaron según los niveles de evidencia (levels of evidence-LoE): tres con LoE 5 y siete con LoE 6. Conclusiones: Existen pocas publicaciones sobre la sistematización de la atención de enfermería perioperatoria relacionada con la seguridad del paciente. Los artículos seleccionados presentan un bajo nivel de evidencia y el enfoque se dirige hacia la seguridad del paciente, y se limita al uso de la lista de verificación y la implementación de la sistematización de la atención de enfermería perioperatoria. Palabras clave: Atención perioperativa. Enfermería perioperativa. Centro quirúrgico. Seguridad del paciente.

'Enfermeira do Centro Cirúrgico Sarmento Barata do Hospital Santa Clara na Irmandade da Santa Casa de Misericórdia de Porto Alegre - Porto Alegre (RS), Brasil.

${ }^{2}$ Professora Colaboradora do Programa de Mestrado Profissional em Enfermagem da Universidade Federal de Ciências da Saúde de Porto Alegre (UFCSPA) - Porto Alegre (RS), Brasil.

3Professora adjunta III da Universidade Federal de Ciências da Saúde de Porto Alegre (UFCSPA) - Porto Alegre (RS), Brasil.

*Autora correspondente: marielli.jost@gmail.com

Recebido: 20/04/2018 - Aprovado: 24/06/2018

DOI: $10.5327 / Z 1414-4425201800040009$ 


\section{INTRODUÇÃO}

A sistematização da assistência de enfermagem (SAE) é um método que visa aprimorar o cuidado prestado pelo enfermeiro ao paciente ${ }^{1}$, buscando proporcionar uma assistência segura e com qualidade, melhorando a comunicação entre as equipes ${ }^{2}$. Com isso, evidencia-se a sua importância para os profissionais de enfermagem, trazendo diversos benefícios ao paciente e à equipe assistencial ${ }^{3}$.

De acordo com a Resolução do Conselho Federal de Enfermagem (COFEn) $n^{\circ} 358 / 2009^{4}$, toda instituição de saúde que presta cuidado profissional de enfermagem deverá utilizar a SAE, permitindo implementar na prática assistencial seus conhecimentos técnico-científicos e de humanização, organizando o trabalho profissional quanto a método, pessoal e instrumentos, tornando possível a operacionalização do processo de enfermagem (PE).

O centro cirúrgico (CC) é uma das unidades mais complexas do hospital, constituído de recursos humanos, materiais, equipamentos e tecnologias de alta complexidade, destinados a atender o paciente cirúrgico no período perioperatório $^{5}$. Os enfermeiros que atuam nessa área podem utilizar a sistematização da assistência de enfermagem perioperatória (SAEP), com o propósito de promover assistência de qualidade ao paciente cirúrgico de forma continuada, participativa, individualizada e documentada. Entende-se como perioperatório todos os períodos que envolvem um ato cirúrgico, a saber: pré-operatório, transoperatório e pós-operatório ${ }^{6}$.

Em 2004, foi lançada a Aliança Mundial para a Segurança do Paciente, pela Organização Mundial da Saúde (OMS), buscando a melhoria da segurança do paciente. A partir dela, determinou-se um desafio global, cujo objetivo era elevar os padrões de qualidade dos serviços de saúde para a realização da cirurgia segura ${ }^{7}$. Nesse sentido, a Joint Commission International $(\mathrm{JCI})^{8}$ promoveu as metas internacionais para segurança do paciente: identificar os pacientes corretamente; melhorar a comunicação efetiva; melhorar a segurança de medicamentos de alta vigilância; assegurar cirurgias com local de intervenção correto, procedimento correto e paciente correto; reduzir o risco de infecções associadas aos cuidados de saúde; e reduzir o risco de lesões ao paciente, decorrentes de quedas.

O Brasil faz parte da Aliança Mundial para a Segurança do Paciente da OMS e, em 2013, o Ministério da Saúde (MS) instituiu o Programa Nacional de Segurança do Paciente (PNSP), por meio da Portaria $\mathrm{n}^{\circ} 529^{9}$, contribuindo para a qualificação do cuidado em saúde. Assim, a Agência Nacional de Vigilância Sanitária (ANVISA) publicou a Resolução da Diretoria Colegiada (RDC) $n^{0} 36 / 2013^{10}$, instituindo as ações para a promoção da segurança do paciente e visando à melhoria da qualidade nas instituições.

Atualmente, as instituições de saúde, objetivando implementar e garantir a qualidade da assistência, utilizam os programas de acreditação, como o da Organização Nacional de Acreditação (ONA), reconhecida como entidade competente para o desenvolvimento do processo hospitalar, coordenada pelo Sistema Brasileiro de Acreditação (SBA), que reúne organizações e serviços de saúde, entidades e instituições acreditadoras em prol da segurança do paciente e da melhoria do atendimento ${ }^{11}$.

Para proporcionar uma assistência de qualidade e segura ao paciente cirúrgico, são necessários o envolvimento e a participação de toda a equipe multiprofissional ${ }^{12}$. Estabelecer um vínculo de comunicação eficaz é fundamental para construir parcerias em prol de uma assistência perioperatória mais qualificada e segura, pois ajuda a reduzir a ocorrência de erros durante os processos assistenciais ${ }^{13,14}$. Portanto, a comunicação efetiva é fundamental para o exercício da profissão na área da saúde ${ }^{15}$.

Tendo em vista a importância da SAEP para a melhoria da qualidade e da segurança do paciente cirúrgico, buscou-se, por meio desta pesquisa, fundamentar e fornecer subsídios para a sua implementação em um CC.

\section{OBJETIVO}

Conhecer a abordagem dos artigos científicos publicados sobre a SAEP relacionada à segurança do paciente.

\section{MÉTODO}

Trata-se de uma revisão integrativa, método de pesquisa que visa sintetizar os resultados obtidos sobre um determinado assunto, contribuindo para o aprofundamento do conhecimento do tema, utilizado nas práticas baseadas em evidências. Esse método é realizado em seis etapas: identificação do tema; definição dos critérios de inclusão e exclusão dos artigos e escolha das bases de dados; identificação dos estudos selecionados; síntese dos estudos; análise e interpretação dos dados coletados; e apresentação dos resultados ${ }^{16}$. 
A questão norteadora da pesquisa foi: qual é a abordagem dos artigos científicos publicados sobre a sistematização da assistência de enfermagem no perioperatório relacionada à segurança do paciente?

As bases de dados eletrônicas utilizadas foram: Biblioteca Virtual em Saúde (BVS), National Library of Medicine (PubMed) e periódicos da Coordenação de Aperfeiçoamento de Pessoal de Nível Superior (CAPES).

Os critérios de inclusão estabelecidos foram: artigos científicos publicados com livre acesso on-line, na íntegra, em português ou espanhol ou inglês, nos últimos 10 anos, de 2008 a 2017. Considerou-se como critérios de exclusão: publicações classificadas como editorial, cartas, dissertações, teses, manuais e protocolos e artigos que não abordassem a questão de pesquisa.

O período de coleta de dados ocorreu de $1^{\circ}$ de novembro a 15 de novembro de 2017, utilizando descritores controlados, obtidos nos Descritores em Ciências da Saúde (DeCS) e no Medical Subject Headings (MeSH): "assistência perioperatória" ("perioperative care"); "segurança do paciente" ("patient safety"); "processo de enfermagem” ("nursing process"); "enfermagem perioperatória" ("perioperative nursing"); e "centro cirúrgico" ("surgical centers"). Também foi utilizado um descritor não controlado: "sistematização da assistência" " "systematization of assistance"). Esses descritores foram combinados entre si pelos operadores booleanos "AND" e/ou "OR", conforme apresentado no Quadro 1.

Quadro 1. Descritores combinados com operadores booleanos utilizados nas etapas de busca dos artigos.

\begin{tabular}{|c|c|c|c|}
\hline $\begin{array}{c}\text { Etapas } \\
\text { da busca }\end{array}$ & $\begin{array}{c}\text { Combinações dos descritores } \\
\text { com operadores booleanos }\end{array}$ \\
\hline $1^{\mathrm{a}}$ & $\begin{array}{c}\text { (enfermagem perioperatória AND } \\
\text { sistematização da assistência) }\end{array}$ \\
\hline $2^{\mathrm{a}}$ & $\begin{array}{c}\text { (centro cirúrgico AND } \\
\text { sistematização da assistência) } \\
3^{\mathrm{a}}\end{array}$ & $\begin{array}{c}\text { (assistência perioperatória AND } \\
\text { processo de enfermagem) }\end{array}$ \\
\hline $4^{\mathrm{a}}$ & $\begin{array}{c}\text { (assistência perioperatória AND } \\
\text { segurança do paciente) }\end{array}$ \\
\hline $5^{\mathrm{a}}$ & $\begin{array}{c}\text { (enfermagem perioperatória OR assistência } \\
\text { perioperatória) AND (sistematização da } \\
\text { assistência OR processo de enfermagem) }\end{array}$ \\
\hline $6^{\mathrm{a}}$ & $\begin{array}{c}\text { (enfermagem perioperatória OR assistência } \\
\text { perioperatória) AND (segurança do paciente OR } \\
\text { processo de enfermagem) }\end{array}$ \\
\hline
\end{tabular}

Os artigos foram avaliados e classificados quanto ao seu rigor científico conforme as características de cada estudo, utilizando os instrumentos de avaliação propostos pela Joanna Briggs Institute $(\mathrm{JBI})^{17}$. Após essa avaliação, foi realizada a classificação por nível de evidência (NE), conforme validade e confiabilidade. Nessa etapa, foi utilizado um instrumento baseado na Rating System for the Hierarchy of Evidence for Intervention/Treatment Question para a classificação do NE dos estudos. Os níveis referem-se:

- a revisões sistemáticas ou metanálises de ensaios clínicos randomizados relevantes (NE 1);

- a um ou mais ensaios clínicos randomizados (NE 2);

- a ensaios clínicos controlados sem randomização (NE 3);

- a casos controle e estudos de coorte (NE 4);

- a revisões sistemáticas de estudos descritivos e estudos qualitativos (NE 5);

- a evidência de um único estudo descritivo ou qualitativo (NE 6);

- a relatórios de opiniões de especialistas $(\mathrm{NE} 7)^{18}$.

\section{RESULTADOS}

Inicialmente, realizou-se a busca nas bases de dados, de acordo com o cruzamento dos descritores citados anteriormente, apresentados na Tabela 1.

$\mathrm{Na}$ busca inicial, realizou-se a leitura dos títulos, sendo selecionados 30 artigos nas bases de dados. Contudo, sete artigos estavam repetidos em diferentes bases. Após realizar a leitura dos resumos e dos artigos na íntegra, foram selecionados 15 que atendiam aos critérios de seleção. Pela avaliação da qualidade interna dos artigos, utilizando os instrumentos de avaliação propostos pela JBI, cinco artigos foram excluídos.

Conforme o seguimento da análise dos artigos selecionados, totalizando 10 , foi possível verificar a quantidade conforme o ano e a distribuição desses, sendo identificados em $2009(\mathrm{n}=1) ; 2011(\mathrm{n}=3) ; 2012(\mathrm{n}=1) ; 2013(\mathrm{n}=2) ; 2014(\mathrm{n}=1)$ e $2015(n=2)$.

Em relação à produção e sua divulgação, identificaram-se oito artigos produzidos e publicados em periódicos nacionais e dois em periódicos internacionais: Ciência, Cuidado e Saúde ( $\mathrm{n}=1)$; Revista de Enfermagem da Universidade Federal de Pernambuco (UFPE) ( $n=1)$; Revista de Administração em Saúde ( $\mathrm{n}=1)$; Revista de Enfermagem da Universidade Federal de Santa Maria (UFSM) (n=1); Revista Gaúcha de 
Enfermagem ( $\mathrm{n}=1)$; Revista Mineira de Enfermagem ( $\mathrm{n}=1)$; Escola Anna Nery Revista de Enfermagem ( $\mathrm{n}=1)$; Revista SOBECC ( $\mathrm{n}=1)$; Online Brazilian Journal of Nursing $(\mathrm{n}=1)$; e Journal Nursing Health $(\mathrm{n}=1)$.

Quanto ao delineamento dos estudos, constatou-se: pesquisa qualitativa $(n=7)$; pesquisa quantitativa $(n=2)$ e pesquisa mista/qualitativa e quantitativa $(\mathrm{n}=1)$. Dessa forma, os estudos foram classificados conforme NE: três com NE 5 e sete com NE 6.

A partir da pesquisa realizada, os artigos foram analisados e agrupados em dois tópicos conforme enfoque: cirurgia segura e segurança do paciente; e SAEP. Os Quadros 2 e 3 apresentam a síntese dos artigos selecionados na revisão integrativa.

Tabela 1. Síntese da busca nas bases de dados. Porto Alegre, 2017. BVS PubMed CAPES

Descritor: (enfermagem perioperatória AND sistematização da assistência)

\begin{tabular}{|l|l|l|l|}
\hline Total de artigos encontrados & 37 & 00 & 04 \\
\hline Total de artigos selecionados & 06 & 00 & 00 \\
\hline
\end{tabular}

Descritor: (centro cirúrgico AND sistematização da assistência)

\begin{tabular}{|l|l|l|l|}
\hline Total de artigos encontrados & 27 & 00 & 22 \\
\hline Total de artigos selecionados & 04 & 00 & 01 \\
\hline
\end{tabular}

Descritor: (assistência perioperatória AND processo de enfermagem)

\begin{tabular}{|l|l|l|l|}
\hline Total de artigos encontrados & 69 & 00 & 03 \\
\hline Total de artigos selecionados & 06 & 00 & 00 \\
\hline
\end{tabular}

Descritor: (assistência perioperatória AND segurança do paciente)

\begin{tabular}{|l|c|c|c|}
\hline Total de artigos encontrados & 198 & 00 & 06 \\
\hline Total de artigos selecionados & 04 & 00 & 00 \\
\hline
\end{tabular}

Descritor: (enfermagem perioperatória OR assistência perioperatória) AND (sistematização da assistência OR processo de enfermagem)

\begin{tabular}{|l|l|l|l|}
\hline Total de artigos encontrados & 37 & 00 & 10 \\
\hline Total de artigos selecionados & 07 & 00 & 00 \\
\hline
\end{tabular}

Descritor: (enfermagem perioperatória OR assistência perioperatória) AND (segurança do paciente OR processo de enfermagem)

\begin{tabular}{|c|c|c|c|}
\hline Total de artigos encontrados & 239 & 00 & 15 \\
\hline Total de artigos selecionados & 02 & 00 & 0 \\
\hline Total após critérios de seleção & $\mathbf{0 9}$ & $\mathbf{0 0}$ & $\mathbf{0 1}$ \\
\hline
\end{tabular}

BVS: Biblioteca Virtual em Saúde; PubMed: National Library of Medicine; CAPES: Coordenação de Aperfeiçoamento de Pessoal de Nível Superior.

\section{DISCUSSÃO}

A análise possibilitou agrupar o tema em dois tópicos, conforme o enfoque dos artigos. No Quadro 2, foram incluídos os artigos com enfoque na cirurgia segura e segurança do paciente, que abordaram a importância da utilização do checklist cirúrgico para a qualidade assistencial.

A OMS 7 lançou o Segundo Desafio Global, que compreende Cirurgias Seguras Salvam Vidas, cujo objetivo é melhorar o cuidado ao paciente cirúrgico, por meio da utilização da lista de verificação cirúrgica como forma de auxiliar a equipe a reduzir a ocorrência de danos ao paciente. Busca melhorar a segurança assistencial e a comunicação da equipe cirúrgica, utilizando um checklist que orienta uma interação verbal, confirmando os padrões de cuidados, minimizando os riscos mais comuns e evitáveis, assim como o bem-estar dos pacientes cirúrgicos ${ }^{19-21,22}$.

Um estudo ${ }^{21}$ avaliou o registro do checklist em cirurgias ortopédicas, que envolvem membros e dupla lateralidade, onde a possibilidade de erro é ainda maior, devendo ser realizada a demarcação do sítio cirúrgico para evitar eventos adversos. Assim, os itens de verificação e o preenchimento correto e efetivo desse instrumento são muito importantes, pois previnem erros e promovem a segurança do paciente no procedimento cirúrgico, e os resultados podem contribuir para o planejamento de ações institucionais corretivas diante dos registros do checklist cirúrgico.

Em outro estudo ${ }^{19}$, os autores ressaltaram a importância das instituições proporcionarem capacitações referentes à utilização do checklist cirúrgico, enfatizando sua importância para que seja aplicado na prática diária, buscando ser viável para garantir cirurgias seguras e contribuir para que o processo de comunicação no ambiente cirúrgico seja efetivo. A realização de capacitação com a equipe estimula a promoção de melhores práticas. Ressalta-se que o checklist deve ser rotina no CC para melhorar a segurança do paciente, portanto o preenchimento correto desse instrumento deve ser um trabalho contínuo ${ }^{22}$.

O checklist deve ser realizado nos três momentos do procedimento anestésico-cirúrgico: antes da indução anestésica, antes do início do procedimento/antes da incisão na pele, e ao final do procedimento/antes da saída do paciente da sala operatória. A implantação do instrumento gera algumas dificuldades no CC, pois, muitas vezes, não há uma boa aceitação da equipe cirúrgica, o que prejudica a sua participação para a aplicação dessa ferramenta. Nesse sentido, o papel do enfermeiro é muito importante, pois contribui com a aplicação 
do checklist e a orientação da equipe na sua utilização, enfatizando os benefícios para os profissionais e os pacientes ${ }^{19}$.

Com isso, estudos evidenciaram que a utilização do checklist melhora a comunicação com a equipe cirúrgica, pois busca melhor interação entre paciente e profissionais, proporcionando assistência de qualidade e segura ${ }^{19,20-22}$.

O Quadro 3 incluiu artigos que abordaram a SAEP, ressaltando a importância da sua implementação no CC, visando à qualidade da assistência prestada.
A SAE contribui para a organização do trabalho da equipe de enfermagem, porém é complexa, por ser diferente em cada setor, sendo executada em quatro etapas do PE: coleta de dados, diagnóstico de enfermagem, planejamento e implementação da assistência de enfermagem ${ }^{27}$. Porém, este estudo ressalta as facilidades e as dificuldades em relação à sua implantação no CC. As facilidades enfatizadas foram que, com a implantação, o enfermeiro passa a conhecer melhor o paciente, realizando um trabalho contínuo de enfermagem,

Quadro 2. Síntese dos artigos com enfoque na cirurgia segura e segurança do paciente.

\begin{tabular}{|c|c|c|c|c|}
\hline $\begin{array}{l}\text { Autores, título } \\
\text { e periódico }\end{array}$ & Objetivos & Delineamento & Principais resultados & $\begin{array}{l}\text { Nível de } \\
\text { evidência }\end{array}$ \\
\hline $\begin{array}{l}\text { Pancieri AP, Santos BP, } \\
\text { Avila MAG, Braga EM }{ }^{19} \\
\text { Checklist de cirurgia } \\
\text { segura: análise } \\
\text { da segurança e } \\
\text { comunicação das equipes } \\
\text { de um hospital escola. } \\
\text { Rev Gaúcha Enferm. } \\
2013 \text {. }\end{array}$ & $\begin{array}{l}\text { Aplicar o checklist } \\
\text { de “cirurgia } \\
\text { segura”, da OMS, } \\
\text { nas especialidades } \\
\text { cirúrgicas de um } \\
\text { hospital escola e } \\
\text { verificar a opinião } \\
\text { das equipes sobre a } \\
\text { influência da aplicação } \\
\text { deste na segurança } \\
\text { do processo cirúrgico } \\
\text { e da comunicação } \\
\text { interpessoal da equipe. }\end{array}$ & $\begin{array}{l}\text { Descritivo, com } \\
\text { abordagem } \\
\text { qualitativa }\end{array}$ & $\begin{array}{l}\text { É preciso que as instituições façam } \\
\text { uso da ferramenta e saibam da sua } \\
\text { importância, garantindo cirurgias seguras e } \\
\text { implementando processos de comunicação } \\
\text { entre as equipes. Ainda se tem dificuldade } \\
\text { na implantação do checklist, no que se } \\
\text { refere à aceitação da equipe cirúrgica. } \\
\text { Os sujeitos do estudo não perceberam } \\
\text { mudanças na comunicação interpessoal } \\
\text { com o uso do checklist, contudo indicaram } \\
\text { que o uso proporcionou mais segurança } \\
\text { ao procedimento e algumas adaptações ao } \\
\text { checklist foram recomendadas. }\end{array}$ & NE 6 \\
\hline $\begin{array}{l}\text { Guzzo GM, Guimarães } \\
\text { SM, Magalhães AMM }{ }^{20} \\
\text { Efeitos e desafios da } \\
\text { implantação de um } \\
\text { sistema de verificação } \\
\text { de segurança cirúrgica: } \\
\text { revisão integrativa. } \\
\text { J Nurs Health. } 2014 \text {. }\end{array}$ & $\begin{array}{l}\text { Identificar os efeitos } \\
\text { do uso de um sistema } \\
\text { de verificação de } \\
\text { segurança cirúrgica, } \\
\text { bem como os desafios } \\
\text { de sua implantação, } \\
\text { disponíveis na } \\
\text { literatura. }\end{array}$ & $\begin{array}{l}\text { Revisão } \\
\text { integrativa }\end{array}$ & $\begin{array}{l}\text { Utilização do sistema de verificação de } \\
\text { segurança cirúrgica traz melhores resultados } \\
\text { assistenciais ao paciente cirúrgico. É } \\
\text { preciso acompanhamento contínuo de } \\
\text { sua implantação para garantir o pleno } \\
\text { funcionamento e aprimorar as práticas no CC } \\
\text { com qualidade e segurança ao paciente. }\end{array}$ & NE 6 \\
\hline $\begin{array}{l}\text { Amaya MR, Maziero ECS, } \\
\text { Grittem L, Cruz EDA }{ }^{21} \\
\text { Análise do registro e } \\
\text { conteúdo de checklists } \\
\text { para cirurgia segura. } \\
\text { Esc Anna Nery. } 2015 \text {. }\end{array}$ & $\begin{array}{l}\text { Analisar e relacionar o } \\
\text { registro de informações } \\
\text { e conteúdo do checklist } \\
\text { com os objetivos do } \\
\text { Programa Cirurgias } \\
\text { Seguras Salvam Vidas. }\end{array}$ & $\begin{array}{c}\text { Pesquisa } \\
\text { quantitativa }\end{array}$ & $\begin{array}{l}\text { Planejar ações para que o checklist seja } \\
\text { registrado corretamente e com a alta adesão } \\
\text { do seu preenchimento. Permitiu identificar } \\
\text { potenciais riscos cirúrgicos, contribuindo } \\
\text { para uma prática profissional focada na } \\
\text { segurança do paciente. }\end{array}$ & NE 5 \\
\hline $\begin{array}{l}\text { Elias ACGP, Schmidt DRC, } \\
\text { Yonekura CSI, Dias AO, Ursi } \\
\text { ES, Silva RPJ, Feijó VBER } \\
\text { Avaliação da adesão } \\
\text { ao checklist de cirurgia } \\
\text { segura em hospital } \\
\text { universitário público. } \\
\text { Rev SOBECC. } 2015 .\end{array}$ & $\begin{array}{l}\text { Avaliar a adesão ao } \\
\text { checklist em cirurgias } \\
\text { realizadas em um } \\
\text { hospital escola público, } \\
\text { bem como identificar o } \\
\text { perfil do paciente com a } \\
\text { sua utilização. }\end{array}$ & $\begin{array}{c}\text { Estudo } \\
\text { descritivo, } \\
\text { qualitativo }\end{array}$ & $\begin{array}{l}0 \text { checklist deve ser inserido dentro da rotina } \\
\text { do CC e ser realizado durante as práticas } \\
\text { diárias, promovendo melhor comunicação } \\
\text { entre as equipes e, assim, ser aprimorado, } \\
\text { contribuindo com a redução dos eventos } \\
\text { adversos dos pacientes cirúrgicos. }\end{array}$ & NE 6 \\
\hline
\end{tabular}


Quadro 3. Síntese dos artigos com enfoque em sistematização da assistência de enfermagem perioperatória.

\begin{tabular}{|c|c|c|c|c|}
\hline $\begin{array}{l}\text { Autores, título } \\
\text { e periódico }\end{array}$ & Objetivos & Delineamento & Principais resultados & $\begin{array}{l}\text { Nível de } \\
\text { evidência }\end{array}$ \\
\hline $\begin{array}{l}\text { Grittem L, Meier MJ, } \\
\text { Peres AM³ } \\
\text { Sistematização } \\
\text { da assistência } \\
\text { perioperatória: uma } \\
\text { pesquisa qualitativa. } \\
\text { Online Braz J Nurs. } 2009 .\end{array}$ & $\begin{array}{l}\text { Desenvolver um } \\
\text { processo participativo } \\
\text { para estruturar } \\
\text { a assistência de } \\
\text { enfermagem } \\
\text { perioperatória na } \\
\text { Unidade de Centro } \\
\text { Cirúrgico de um } \\
\text { hospital de Curitiba. }\end{array}$ & $\begin{array}{c}\text { Estudo } \\
\text { qualitativo }\end{array}$ & $\begin{array}{l}\text { Os argumentos possibilitaram refletir sobre a } \\
\text { assistência de enfermagem perioperatória e } \\
\text { a valorização das atividades executadas pela } \\
\text { enfermeira como forma de colaborar para o } \\
\text { seu reconhecimento profissional. }\end{array}$ & NE 6 \\
\hline $\begin{array}{l}\text { Gonçalves RMDA, Pereira } \\
\text { MER, Pedrosa LAK, Silva } \\
\text { QCG, Abreu RMD24 } \\
\text { A comunicação verbal } \\
\text { enfermeiro-paciente } \\
\text { no perioperatório de } \\
\text { cirurgia cardíaca. } \\
\text { Cienc Cuid Saúde. } 2011 .\end{array}$ & $\begin{array}{c}\text { Verificar a importância } \\
\text { da comunicação verbal } \\
\text { do } \\
\text { enfermeiro com } \\
\text { o paciente no } \\
\text { perioperatório de } \\
\text { cirurgia cardíaca em um } \\
\text { hospital universitário. }\end{array}$ & $\begin{array}{l}\text { Descritivo- } \\
\text { exploratório, } \\
\text { quantitativa } \\
\text { e qualitativa } \\
\text { (misto) }\end{array}$ & $\begin{array}{l}\text { Identificou a importância da comunicação } \\
\text { efetiva do enfermeiro, buscando ótima } \\
\text { interação com o paciente e a equipe, } \\
\text { identificando suas necessidades e elaborando } \\
\text { ações sistematizadas, essencial para a } \\
\text { qualidade do cuidado e a sistematização da } \\
\text { assistência perioperatória. }\end{array}$ & NE 5 \\
\hline $\begin{array}{l}\text { Umann J, Guido LA, } \\
\text { Linch GFC, Freitas EO }{ }^{25} \\
\text { Enfermagem } \\
\text { perioperatória em } \\
\text { cirurgia cardíaca: revisão } \\
\text { integrativa da literatura. } \\
\text { Rev Min Enferm. } 2011 .\end{array}$ & $\begin{array}{c}\text { Investigar as } \\
\text { produções científicas } \\
\text { sobre a assistência } \\
\text { perioperatória } \\
\text { de enfermagem ao } \\
\text { paciente em cirurgia } \\
\text { cardíaca. }\end{array}$ & $\begin{array}{l}\text { Revisão } \\
\text { integrativa }\end{array}$ & $\begin{array}{l}\text { Torna-se importante a realização de } \\
\text { um plano assistencial e protocolos de } \\
\text { intervenções, devendo-se implementar o PE. } \\
\text { Deve-se realizar pesquisas que comprovem a } \\
\text { eficácia das intervenções, para que auxiliem } \\
\text { no avanço cientíico da profissão com a } \\
\text { implementação de práticas baseadas em } \\
\text { evidências. }\end{array}$ & NE 6 \\
\hline $\begin{array}{l}\text { Klein AGS, Bitencourt } \\
\text { JVOV, Dal Pai D, } \\
\text { Wegner W }{ }^{26} \\
\text { Registros de } \\
\text { enfermagem no período } \\
\text { perioperatório. } \\
\text { Rev Enferm UFPE } \\
\text { Online. } 2011 .\end{array}$ & $\begin{array}{l}\text { Avaliar os registros } \\
\text { de enfermagem no } \\
\text { período perioperatório } \\
\text { de um hospital de Porto } \\
\text { Alegre, Rio Grande do } \\
\text { Sul. }\end{array}$ & $\begin{array}{l}\text { Estudo } \\
\text { quantitativo, } \\
\text { com abordagem } \\
\text { descritiva. }\end{array}$ & $\begin{array}{l}\text { Verifica-se uma falta e dificuldade dos } \\
\text { profissionais da enfermagem em realizar } \\
\text { os registros no perioperatório, o que } \\
\text { compromete o PE. Recomenda-se elaboração } \\
\text { de um instrumento único e sistematizado } \\
\text { para os registros do perioperatório, } \\
\text { propiciando um fluxo de informações sobre } \\
\text { o procedimento anestésico-cirúrgico. Deverá } \\
\text { ter motivação e empenho de toda equipe para } \\
\text { o desenvolvimento e a implementação de } \\
\text { instrumentos específicos que possam ser de } \\
\text { benefício ao paciente cirúrgico. }\end{array}$ & NE 5 \\
\hline $\begin{array}{l}\text { Adamy EK, Tosatti M }{ }^{27} \\
\text { Sistematização } \\
\text { da assistência de } \\
\text { enfermagem no período } \\
\text { perioperatório: visão da } \\
\text { equipe de enfermagem. } \\
\text { Rev Enferm UFSM. } \\
2012 \text {. }\end{array}$ & $\begin{array}{l}\text { Avaliar a implantação } \\
\text { da SAE no período } \\
\text { perioperatório de um } \\
\text { hospital do oeste de } \\
\text { Santa Catarina, sob } \\
\text { a visão da equipe de } \\
\text { enfermagem. }\end{array}$ & $\begin{array}{l}\text { Qualitativo, } \\
\text { descritivo. }\end{array}$ & $\begin{array}{l}\text { Aponta algumas dificuldades em relação } \\
\text { à implantação da SAE, como falta de } \\
\text { comprometimento coletivo, implantação } \\
\text { rápida, sem capacitação adequada da } \\
\text { equipe de enfermagem, sem ter um sistema } \\
\text { informatizado para favorecer o processo de } \\
\text { trabalho e falta de pessoal. Ressaltaram a } \\
\text { importância da implantação da SAE em relação } \\
\text { à segurança do paciente cirúrgico, os registros } \\
\text { se tornaram indispensáveis e facilitou o acesso } \\
\text { às informações dos pacientes, mantendo } \\
\text { um trabalho organizado e a continuidade da } \\
\text { assistência. Compete à instituição oportunizar } \\
\text { estratégias que resultem para uma SAE efetiva } \\
\text { e com ótimos resultados. }\end{array}$ & NE 6 \\
\hline
\end{tabular}


Quadro 3. Continuação.

\begin{tabular}{|l|c|c|c|}
\hline $\begin{array}{l}\text { Autores, título } \\
\text { e periódico }\end{array}$ & Objetivos & Delineamento & $\begin{array}{c}\text { Nível de } \\
\text { evidência }\end{array}$ \\
\hline Santos MC, Rennó CSN ${ }^{28}$ & Principais resultados \\
\hline $\begin{array}{l}\text { Indicadores de qualidade } \\
\text { da assistência de } \\
\text { enfermagem em centro } \\
\text { cirúrgico: revisão } \\
\text { integrativa da literatura. }\end{array}$ & $\begin{array}{c}\text { Identificar os } \\
\text { indicadores de } \\
\text { qualidade da } \\
\text { assistência de } \\
\text { enfermagem em CC. }\end{array}$ & $\begin{array}{c}\text { Revisão } \\
\text { integrativa. }\end{array}$ & $\begin{array}{c}\text { Os enfermeiros do CC devem monitorar e } \\
\text { analisar os indicadores e promover uma } \\
\text { comunicação entre as equipes, bem como o } \\
\text { planejamento das atividades, promovendo } \\
\text { efetividade nos processos de enfermagem. A } \\
\text { SAEP permite ao enfermeiro }\end{array}$ \\
\hline Rev Adm Saúde. 2013. & qualificar a assistência prestada no CC. \\
\hline
\end{tabular}

NE: nível de evidência; CC: centro cirúrgico; SAE: sistematização da assistência de enfermagem; PE: processo de enfermagem; SAEP: sistematização da assistência de enfermagem perioperatória.

acompanhando o paciente cirúrgico de perto, proporcionando uma melhora na assistência prestada e estabelecendo uma rotina. Em relação às dificuldades, foram apontadas a falta de tempo e pessoal para executar a SAE, as mudanças na prática de trabalho e a falta de conhecimento para realizar a sistematização ${ }^{27}$.

Pesquisas $^{23-27}$ ressaltam que os enfermeiros relatam a importância e a necessidade de realizar a SAEP na prática, porém não a executam, devido às várias dificuldades e ao não entendimento para a sua aplicação. Um estudo ${ }^{23}$ relata que o enfermeiro de CC não consegue prestar assistência direta ao paciente no período perioperatório, pois desempenha várias atividades gerenciais no seu trabalho, tendo sobrecarga de atividades, o que torna necessário um planejamento da assistência no qual as tarefas devem ser redistribuídas para que o enfermeiro possa acompanhar o paciente e promover um cuidado integrado.

A SAE possibilita um cuidado humanizado e individualizado, tornando-se importante o uso de indicadores de qualidade da assistência de enfermagem no CC, identificando as intervenções de enfermagem de acordo com as necessidades de cada paciente, realizando um planejamento de suas atividades $^{28}$. Destaca-se a importância de se ter um sistema informatizado na instituição, desenvolvendo um trabalho organizado, realizando os registros adequadamente, otimizando o tempo e facilitando o acesso às informações dos pacientes ${ }^{27}$. Os registros de enfermagem devem ser realizados no período perioperatório em um instrumento sistematizado, buscando confirmar a prática e os cuidados realizados aos pacientes, de modo a possibilitar o fluxo das informações no procedimento anestésico-cirúrgico ${ }^{26}$.

A SAEP permite ao enfermeiro do CC qualificar a assistência a ser dispensada aos pacientes no período perioperatório, planejando a assistência, promovendo melhor comunicação entre as equipes, monitorando e analisando os indicadores para propiciar efetividade dos processos de enfermagem ${ }^{28}$.

Estudos ${ }^{2426}$ ressaltam a importância da comunicação entre as equipes, a fim de contribuir para a melhoria da assistência perioperatória, desenvolvendo ações que visam orientar e avaliar as necessidades de cada paciente cirúrgico, resultando em um planejamento dos cuidados com qualidade durante todo o processo cirúrgico.

\section{CONSIDERAÇÕES FINAIS}

Este artigo buscou conhecer a abordagem dos artigos científicos, considerando-se poucas as publicações sobre a SAEP relacionada à segurança do paciente cirúrgico. Os artigos selecionados apresentam baixo NE e a abordagem direciona-se à segurança do paciente, restringindo-se à utilização do checklist e à implementação da SAEP.

Os resultados deste estudo evidenciam a importância da utilização do checklist no CC, de forma a proporcionar maior segurança ao paciente no procedimento anestésico-cirúrgico, desenvolvendo melhor interação entre o paciente e a equipe, melhorando, assim, a comunicação na linha do cuidado perioperatório. Os resultados demonstraram que a implementação da SAEP possibilita a interação do enfermeiro no processo perioperatório, planejando a assistência de acordo com as necessidades de cada paciente, visando à qualidade da assistência prestada, focada num processo com cunho científico, embasando todas as práticas adotadas. Sugere-se a construção de mais pesquisas que envolvam essa temática para qualificar a assistência de enfermagem ao paciente no período perioperatório. 


\section{REFERÊNCIAS}

1. Viana VO, Pires PS. Validação de instrumento de sistematização da assistência de enfermagem. Rev Enferm Atenção Saúde. 2014;3(2):64-75. https://doi.org/10.18554/

2. Lourenção DCA, Tronchin DMR. Segurança do paciente no ambiente cirúrgico: tradução e adaptação cultural de instrumento validado. Acta Paul Enferm. 2016;29(1):1-8. http://dx.doi. org/10.1590/1982-0194201600002

3. Ferrari D, Costa AEK, Pissaia LF, Moreschi C. A visão da equipe de enfermagem sobre a Sistematização da Assistência de Enfermagem em um hospital de médio porte. Cad Pedagógico. 2016;13(3):109-16. http://dx.doi.org/10.22410/issn.1983-0882.v13i2a2016.1160

4. Conselho Federal de Enfermagem. Resolução n 358, de 15 de outubro de 2009. Dispõe sobre a Sistematização da Assistência de Enfermagem e a implementação do Processo de Enfermagem em ambientes, públicos ou privados, em que ocorre o cuidado profissional de enfermagem, e dá outras providências. Brasília: COFEN; 2009.

5. Carvalho R, Bianchi ERF, eds. Enfermagem em centro cirúrgico e recuperação. 2a ed. Barueri: Manole; 2016.

6. Associação Brasileira de Enfermeiros de Centro Cirúrgico, Recuperação Anestésica e Centro de Material e Esterilização. Diretrizes de práticas em enfermagem cirúrgica e processamento de produtos para a saúde. $7^{a}$ ed. São Paulo: SOBECC/Barueri: Manole; 2017.

7. Organização Mundial da Saúde. Segundo desafio global para a segurança do paciente: manual - cirurgias seguras salvam vidas (orientações para cirurgia segura da OMS). Rio de Janeiro: Organização Pan-Americana da Saúde, Ministério da Saúde, Agência Nacional de Vigilância Sanitária; 2009.

8. Joint Commission Intermational. Manual de padrões de acreditação da Joint Commission International para hospitais. Estados Unidos: Joint Commission International; 2011.

9. Brasil. Ministério da Saúde. Portaria n 529, de $1^{\circ}$ de abril de 2013. Institui o Programa Nacional de Segurança do Paciente (PNSP). Brasília: Ministério da Saúde; 2013.

10. Brasil. Ministério da Saúde. Agência Nacional de Vigilância Sanitária. Resolução da Diretoria Colegiada-RDC n³6, de 25 de julho de 2013. Institui ações para a segurança do paciente em serviços de saúde e dá outras providências. Brasília: ANVISA; 2013.

11. Organização Nacional de Acreditação. 0 que é acreditação [Internet]. 2017 [acessado em $1^{\circ}$ out. 2017]. Disponível em: http://www.ona.org.br/

12. Henriques AHB, Costa SS, Lacerda LS. Assistência de enfermagem na segurança do paciente cirúrgico: revisão integrativa. Cogitare Enferm. 2016;21(4):1-9.

13. Martins FZ, Dall'Agnol CM. Centro cirúrgico: desafios e estratégias do enfermeiro nas atividades gerenciais. Rev Gaúcha Enferm. 2016;37(4):e56945. http://dx.doi.org/10.1590/1983-1447.2016.04.56945

14. Miranda AL, Carvalho AKO, Lopes AAS, Oliveira VRC, Carvalho PMG, Carvalho HEF. Contribuição da enfermagem à segurança do paciente: revisão integrativa. Rev Sonare. 2017;16(1):109-17.
15. Negri EC, Mazzo A, Martins JCA, Pereira Jr. GA, Almeida RGS, Pedersoli CE. Simulação clínica com dramatização: ganhos percebidos por estudantes e profissionais de saúde. Rev Latino-am Enferm. 2017;25:e2916. http://dx.doi.org/10.1590/1518-8345.1807.2916

16. Cunha PLP, ed. Manual revisão bibliográfica sistemática integrativa: a pesquisa baseada em evidências. Belo Horizonte: Grupo Ânima; 2014.

17. Vernaya M, McAdam J. The effectiveness of probiotics in reducing the incidence of Clostridium difficile associated diarrhea in elderly patients: a systematic review protocol. JBI Database System Rev Implement Rep. 2015;13(8):79-91. https://doi.org/10.11124/jbisrir-2015-2197

18. Melnyk BM, Fineout-Overholt E. Evidence-based practice in nursing $\&$ healthcare: a guide to best practice. $2^{\mathrm{a}}$ ed. Filadélfia: Lippincott Williams \& Wilkins; 2011.

19. Pancieri AP, Santos BP, Avila MAG, Braga EM. Checklist de cirurgia segura: análise da segurança e comunicação das equipes de um hospital escola. Rev Gaúcha Enferm. 2013;34(1):71-8. http://dx.doi. org/10.1590/S1983-14472013000100009

20. Guzzo GM, Guimarães SM, Magalhães AMM. Efeitos e desafios da implantação de um sistema de verificação de segurança cirúrgica: revisão integrativa. J Nurs Health. 2014;4(2):155-64. http://dx.doi. org/10.15210/jonah.v4i2.3393

21. Amaya MR, Maziero ECS, Grittem L, Cruz EDA. Análise do registro e conteúdo de checklists para cirurgia segura. Esc Anna Nery. 2015;19(2):246-51. http://dx.doi.org/10.5935/1414-8145.20150032

22. Elias ACGP, Schmidt DRC, Yonekura CSI, Dias AO, Ursi ES, Silva RPJ, et al. Avaliação da adesão ao checklist de cirurgia segura em hospital universitário público. Rev SOBECC. 2015;20(3):128-33. http://dx.doi. org/10.5327/Z1414-4425201500030002

23. Grittem L, Meier MJ, Peres AM. Sistematização da assistência perioperatória: uma pesquisa qualitativa. Online Braz J Nurs. 2009;8(3):65.

24. Gonçalves RMDA, Pereira MER, Pedrosa LAK, Silva QCG, Abreu RMD. A comunicação verbal enfermeiro-paciente no perioperatório de cirurgia cardíaca. Cienc Cuid Saúde. 2011;10(1):27-34. http://dx.doi. org/10.4025/cienccuidsaude.v10i1.8681

25. Umann J, Guido LA, Linch GFC, Freitas EO. Enfermagem perioperatória em cirurgia cardíaca: revisão integrativa da literatura. Rev Min Enferm. 2011;15(2):275-81. http://www.dx.doi.org/S1415-27622011000200017

26. Klein AGS, Bitencourt JVOV, Dal Pai D, Wegner W. Registros de enfermagem no período perioperatório. Rev Enferm UFPE Online. 2011;5(5):1096-104. http://dx.doi.org/10.5205/ reuol.1302-9310-2-LE.0505201103

27. Adamy EK, Tosatti M. Sistematização da assistência de enfermagem no período perioperatório: visão da equipe de enfermagem. Rev Enferm UFSM. 2012;2(2):300-10. http://dx.doi.org/10.5902/217976925054

28. Santos MC, Rennó CSN. Indicadores de qualidade da assistência de enfermagem em centro cirúrgico: revisão integrativa da literatura. Rev Adm Saúde. 2013;15(58):27-36. 